\title{
The Most Important Educational Interventions for Reducing Occupational Stress Among Nurses Working in Health Centres: A Systematic Review Study
}

INTRODUCTION: Occupational stress is a very common feature of modern life and is increasing and has a bad effect on cardiovascular systems. Today it is known that stress is associated with many physical and mental illnesses or contributes to its development. This systematic review was conducted with the aim of determining The most important educational interventions for reducing occupational stress among nurses working in health centres.

MATERIALS AND METHODS: In this systematic review, in order to achieve the goal of the study and to improve the accuracy of its study and its comprehension, this integrated overview study was conducted based on the Broome method. The method is done in the form of three steps in the search of texts, data evaluation and data analysis, So, in the search phase, post-retention studies texts are examined in four stages in terms of inclusion criteria. After obtaining the conditions for entry into the study, the content of the study is evaluated and at the end the analysis of the data was done. It was conducted in English and Persian by searching articles in search engines, sites and authoritative scientific databases Google Scholar, Embase, Researchgate, Sciencedirect, PubMed, Springer. In the first stage, 41 papers were found. Of these, 10 related articles that have been published in the last 20 years have been reviewed.

RESULTS: In this study, ten articles were reviewed that show the positive effect of training on reducing the stress of nurses. One of these studies that was on the nurses in the direction of impact of stress management training program on stress and work-life conflict showed that the stress level in the intervention group was significantly lower than the control group. Also, in the intervention group itself, the work stress was reduced compared to before the intervention. The results of the intervention showed that, by reducing work stress, the work-life conflict scores also decreased and there is a significant positive correlation between stress management and work-life conflicts in nurses.

CONCLUSION: Studies have shown that the implementation of work-related stress management training programs can reduce employee stress by increasing the level of employees' knowledge about how to manage occupational stress, how to better manage time and also to modify work interactions to reduce job stress.

KEYWORDS: Occupational stress, Nurses, Health centres

\begin{abstract}
INTRODUCTION
Job stress is the annoying emotional response that occurs in the event of a mismatch between the demands and requirements of a talented job, resources or staffing needs. The importance of stress is not only due to its economic damage, but also its negative effects on the physical and mental health of individuals. Today, it has been determined that stress is associated with many physical and psychological illnesses, or in the creation, development and spread of it has a role. ${ }^{1-3}$ Social and psychological factors not only affect the quality of life, but also often play a role in determining the outcome of coping with a chronic disease. ${ }^{4}$ Stress along with many illnesses will cause many complications, for example, stress with diabetes will increase blood sugar and further complications of diabetes. Diabetes, a chronic and metabolic disease, has always been interacting with stress..$^{-11}$ Complications of diabetes are very common among people with this condition and they will be heavily stressed. ${ }^{12-20}$
\end{abstract}

Job stress is one of the most common features of modern life and is increasing and has a bad effect on cardiovascular systems. Nowadays, it is known that stress is associated with many physical and mental illnesses, or in the creation, transformation and Its expansion is contributing, and the next is the human interest in studying stress, the relationship between stress and performance. ${ }^{21-29}$ Excessive stress impairs performance and reduces the productivity of employees in organizations. Therefore, stress is one of the issues that is being studied in the field of management and organizational behavior, Therefore, organizations in their program pay much attention to the study, control and reduction of stress in the work environment in order to reduce the cost of physical and psychological stress and reduce the productivity of their employees. ${ }^{1}$ This systematic review was conducted with the aim of determining The most important educational interventions for reducing occupational stress among nurses working in health 
centres.

\section{MATERIALS AND METHODS}

In this systematic review using articles published in the last 20 years about the most important educational interventions for reducing occupational stress among nurses. It was conducted in English and Persian by searching articles in search engines, sites and authoritative scientific databases Google Scholar, Embase, Sciencedirect, PubMed, Springer. In the first stage, 41 papers were found. Of these, 10 related articles that have been published in the last 20 years have been reviewed.

In order to achieve the goal of the study and to improve the accuracy of its study and its comprehension, this integrated overview study was conducted based on the Broome method. The method is done in the form of three steps in the search of texts, data evaluation and data analysis, So, in the search phase, post-retention studies texts are examined in four stages in terms of inclusion criteria. After obtaining the conditions for entry into the study, the content of the study is evaluated and at the end the analysis of the data was done.

The criteria for entry of articles include studies published in Persian and in English, Access to their full text was possible, they were published in the last 20 years and Exit criteria include unnamed and nonscientific studies as well as articles that lacked the full text of the article.

To achieve relevant studies, a wide range of keywords including occupational stress, nurses and health centers was used as a one-to-one search, combined with the method "And" and "OR".

\section{RESULTS}

Job stress is the annoying emotional response that occurs in the event of a mismatch between the demands and requirements of a talented job, resources or staffing needs. The importance of stress is not only due to its economic damage, but also its negative effects on the physical and mental health of individuals. Today, it has been determined that stress is associated with many physical and psychological illnesses, or in the creation, development and spread of it has a role. ${ }^{1-3}$ This systematic review was conducted with the aim of determining the most important educational interventions for reducing occupational stress among nurses working in health centres.

In the study, the results of the intervention showed that by reducing work stress, the scores of work-life conflict also decreased ${ }^{30}$, as well as in another study ${ }^{31}$, in the study of the relationship between work stress and labor conflict - Life, there was a significant positive relationship between work stress and worklife conflict. Also, another study from the ${ }^{32}$ study on nurses showed that training in the test group caused a significant reduction in the stress score, but in the control group, this difference was not observed. Also, comparing the scores of both groups before and after the intervention showed a significant difference after the intervention, indicating a decrease in the stress level of the subjects in the intervention group. A further study showed that the average stress of legal students who received four weeks of stress relief training received $6.6 \%$ of the post-intervention phase and $4.6 \%$ at the follow-up stage. 33

In another study, the effect of educational program on functional relaxation based on PRESED model on nurses' anxiety was studied. The relaxation techniques applied were used as objective behaviors, which were taught using short-term lectures with questions and answers, group discussions, practice shows and pamphlets. After the end of the seven training sessions, the call was made through a short message for a month and the presence of a coach. Findings of the study after 6 months showed a significant difference between the mean of hidden and obvious anxiety scores in the intervention group compared to the intervention group before intervention. ${ }^{34}$

In one study, the mean of stress before and one month after intervention in the experimental group showed a significant difference. Also, there was a significant difference between the mean of occupational stress and occupation in the life before, after intervention and intervention in the two groups. ${ }^{35}$

The study to investigate the effect of critical thinking training on job stress on nurses showed a significant decrease in job stress score in group education students, and these results were also significant in 
comparison with control group..$^{36}$

In another study, which was quoted in a study ${ }^{32}$, in order to investigate the effect of concentration-based exercises and deep breathing exercises to reduce stress symptoms in a nurse, the initiative of this study was to measure serum cortisol levels before and after intervention Was investigated. The results showed that the positive effect of training on the stress level of work in the experimental group and the comparison of the scores of the test group was significant in comparison with the control group.

In one study ${ }^{37}$, the effect of education on the basis of stress relief model in emergency department and trauma nurses was performed. The results showed that reducing stress due to exposure to stress materials and stressful conditions in the test group. The knowledge and knowledge of nurses in the intervention group also increased a priori and the results of this study were consistent with similar studies in the field of education, occupational stress and nature of nursing in order to reduce stress..$^{38,39}$

\section{DISCUSSION}

Neuropsychiatric pressure as the most important cause of mental, physical and behavioural diseases of human beings has attracted the attention of physicians, psychologists and scientists of behavioural and management sciences. The importance of stress is not only due to its economic damage, but also its negative effects on the physical and mental health of individuals. Job stress is the annoying emotional response that occurs in the event of a mismatch between the demands and requirements of a talented job, resources or staffing needs. This systematic review was conducted with the aim of determining the most important educational interventions for reducing occupational stress among nurses working in health centres.

Some researchers focus on stress-related work stress in these prominent jobs and some of the individual's individual situation. Regarding the relatively high prevalence of stress and early onset of failure, early diagnosis and control of the condition of the disorder begins and emphasizes. The use of preventive methods, including educational methods, is important in identifying stress in the early stages and coping with $i^{42}$, And stress management training programs increase the ability of individuals to reduce stress and adapt to stress conditions. ${ }^{43}$ In recent years, there have been some moves to address the effects of psychological treatments on reducing occupational stress, and some job counsellors, psychologists, psychotherapists, and psychiatrists use counselling techniques in counselling clinics. One of the ways to control stress and anxiety is to teach stress management programs that give people the right ways to deal with stress and ways to overcome it. 44,45

Therefore, the use of appropriate instructional theory (as a basis for educational work) helps these people recognize stress symptoms and deal with the first signs of stress. ${ }^{46}$ According to study ${ }^{30}$, the use of stress management skills in work life and family life can be assisted with better nurse management time, work stress and fitness, proper diet, the right way to work in the family and the workplace to balance work and life.

\section{CONCLUSION}

Studies have shown that the implementation of workrelated stress management training programs can reduce employee stress by increasing the level of employees' knowledge about how to manage occupational stress, how to better manage time and also to modify work interactions to reduce job stress.

Acknowledgment: The authors of this article is grateful to all those who have collaborated with me in this article.

\section{REFERENCES}

1. Vosoughi NA, Rohollahi A, Mohamad HH. The effect of job stress on general health and job performance on air traffic controllers (ATC).2016; 4757.

2. Khosravi M. Examine job stressors librarians working in academic libraries Ministry of Science, Research and Technology, Tehran, from their perspective, research projects, Information and Documentation Center of Iran, Tehran, 2004.

3. Rhodes J, Smith B. Combat stress. Marine Corps Reference, 2000: p.16-40.

4. Amiri M, Aghaei A, Abedi A, Safari Y. The effect of stress inoculation training on depressive symptoms. Diabetes. 2014; 16-30.

5. Moslemirad M, Madmoli M, Madmoli Y, Niksefat M. Prevalence of type 1 and type 2 diabetes and its related factors in diabetic patients hospitalized in 
Khatam-ol-Anbia hospital in Shoushtar, 2014-15: A retrospective study. Journal of Research in Medical and Dental Science. 2018;6(3):421-6

6. Madmoli M, Eilami O, Rezaie K, Aliabad MA, Moslemirad M. Diabetes and the risk of suffering cardiovascular Diseases: A two-year retrospective study. International Journal of Ecosystems and Ecology Science (IJEES). 2018;8(3): 649-56.

7. Madmoli M Rostami F, Mirsami Yazdi N, Mosavi A, Baraz Sh. Evaluation of Prevalence of Diabetic Foot Ulcer and Its Related Factors in Diabetic Patients Admitted to KHatam-ol-Anbia Hospital in Shoushtar During 2015-2016: A Retrospective Study. International Journal of Ecosystems and Ecology Science (IJEES). 2018; 8 (3): 545-52.

8. Rostami F, Madmoli M, Mirsami Yazdi N, Baraz Sh. Evaluation of The Prevalence of Lower Limb Amputation and Its Related Factors in Diabetic Patients Admitted to KHatam-ol-Anbia Hospital in Shoushtar During The 2015-2016: A Retrospective Study. International Journal of Ecosystems and Ecology Science (IJEES). 2018;8(3):553-6o.

9. Raisifar Z, Afshar Nia A, Madmoli M, Madmoli Y. The Relationship Between Using Insulin and Suffering Alzheimer's Disease in Patients with Diabetes: A Two-Year Study. International Journal of Ecosystems and Ecology Science (IJEES). 2018;8(3): 623-28.

10. Raisifar Z, Afshar Nia A, Maghamesi Moarrefi H, Madmoli M. Evaluation of Gi Bleeding Prevalence and Its Related Factors in Diabetic Patients Hospitalized in KHatam-ol-Anbia Hospital During 2015-16: A Retrospective Study. International Journal of Ecosystems and Ecology Science (IJEES). 2018;8 (3): 6o9-14.

11. Madmoli M, Kord Z, Bandani A, Sedighi N, Rezaei Shandiz M, Darabiyan P, AfsharNia A. Epidemiological and clinical study of patients with Alzheimer's in Five Cities of Khuzestan Province in 2016-2018. Medical Science 2019;23(95):1-5

12. Madmoli M, Fallah bagher shaidaei M, Rohani A, Darabiyan P, Mobarez F. The correlation between alcohol consumption and reducing the age of cancer incidence in patients with this disease. Medical Science 2019;23(95):48-53.

13. Madmoli M, Mahmoudi Dehcheshmeh Z, Rafi A, Zahra Kord, Fariba Mobarez, Pouriya Darabiyan. The rate of some complications and risk factors of diabetes in diabetic patients: Study on cases of 3218 diabetic patients. Medical Science 2019; 3(95):63-8.

14. Mostafa Madmoli, Mehran Yarbig, Negin Sedighi, Pouriya Darabiyan, Fariba Mobarez. Communication between body mass index and the risk of obesity- related cancer: A 5-year study on patients with cancer. Medical Science 2019;23(95):69-74.

15. Madmoli M, Madmoli Y, Khodadadi M, Samsamipour M. Some Factors Affecting Quality of Life in Patients with Diabetes: A systematic Review. Annals of Microbiology and Infectious Diseases. 2019; 2(1);26-30.

16. Madmoli M. Clinical and Laboratory Findingin Children with Leukemia: a Systematic Review. International Journal of Research Studies in Science, Engineering and Technology, 2018;5(10):1-6.

17. Madmoli M. Evaluation of Chemotherapy Complications in Patients with Cancer: A systematic Review. International Journal of Research Studies in Science, Engineering and Technology 2018;5(12):5964 .

18. Madmoli M. Quality of Life in Patients with Cancer and Some Factors Affecting it: A Systematic Review. International Journal of Research Studies in Science, Engineering and Technology. 2019;6(1): 1-7.

19. Madmoli M, Madmoli Y, Khodadadi M, Samsamipour M. Study of Some Effective Treatments for Accelerating Diabetic Foot Ulcer Healing: A Systematic Review. International Journal of Research Studies in Science, Engineering and Technology, 6(2), 2019;34-9.

20. Madmoli M, Madmoli Y, Khodadadi M, Samsamipour M. Factors Affecting the Level of Glycosylated Hemoglobin in Patients with Diabetes: A Systematic Review. Annals of Microbiology and Infectious Diseases 2019;2(1):43-7.

21. Gheisari Z, Beiranvand R, Karimi A, Ghalavandi S, Soleymani A, Madmoli M, Bavarsad AH. Relationship between Occupational Stress and Cardiovascular Risk Factors Determination: A Case-control Study. Journal of Research in Medical and Dental Science. 2018 17;6(3):287-93.

22. Madmoli M, Nikpay S. An Investigation of the Relationship between Spiritual Health and Depression, Anxiety, and Stress among Students of Ilam University of Medical Sciences. Journal of Research in Medical and Dental Science. 2018 17;6(3):294-300.

23. Madmoli Y, Akhaghi Dezfuli SM, Beiranvand R, Saberi Pour B, Azami M, Madmoli M. An epidemiological and clinical survey of patients with $\beta$-thalassemia in dezful in 2015. Iranian Journal of Epidemiology. 2017;13(2):145-52.

24. Mashali H, Toleideh F, Rahmani R, Darabiyan P, Madmoli M. The predictive role of Hyperlipidemia in the incidence of ACS in patients referring to Shahidzadeh Hospital in Behbahan in 2016-2017. Medical Science 2018;22(94):566-70. 
25. Madmoli Y, Madmoli M, Qashqaei Nezhad N, Bosak S. Prevalence of depression and associated factors in adolescents of Masjed-Soleyman. Iranian Journal of Pediatric Nursing. 2016;2(4):22-6.

26. Roughani A, Madmoli M, Raisifar Z, Kikhavani S, Yasemi M, Azami M, Sharifi Z. The prevalence of behavioral disorders and its related factors in elementary school children in Ilam City in 2011-2012. Indian Journal of Forensic Medicine \& Toxicology. 2018;12(4):165-9.

27. Adavi A, Hamid N, Attari Y, Madmoli Y, Madmoli M. Study of the Effect of Problem-Solving Skills Training on Creactivity and Assertiveness Among High School Students. Iranian Journal of Nursing Research. 2016;11(5):48-55.

28. Madmoli M, Madmoli Y, Bigdeli Shamloo MB, Etebari A, Mahmoodi Kouhi A, Azami M. The Relationship Between Depression and Religiousness in Female High School Students of Masjed Soleyman in 2015. Journal of Pediatric Nursing. 2017;3(4):15-22.

29. Madmoli Y, Aslani A, Ahmadi Y, Mousavi M, Mashalchi H, Niksefat M, Madmoli M. Study Habits and Related Factors in Students of Nursing and Midwifery College of Dezful University of Medical Sciences in 2015. Iranian Journal of Nursing Research (IJNR). 2017;12(4):27-33

30. Makie VV. Stress and coping strategies amongst registered nurses working in a South African tertiary hospital: University of the Western Cape; 2006.

31. Chuang Y, Lei Y. Retail staffs working stress and work-family conflict under shifts: Work system study. BAI Conference Papers 2011.

32. Hazavehei SM, Moghadam K, Melika S, Bagheri Kholenjani F, Ebrahimi $H$. The influence of educational interventions to reduce occupational stress: A systematic review. Health and Safety at Work. 2017;7(4):363-74.

33. Sheehy R, Horan JJ. Effects of Stress Inoculation Training for 1st-Year Law Students. International Journal of Stress Management. 2004;11(1):41.

34. Ebrahimi Iraqi Nezhad Z, Tol A, Shojaeezadeh D, Khorsandi M, Bagheri F. Effectiveness of PRECEDE model for health education on anxiety of nurses employed in hospitals of Arak University of Medical Sciences: application of PRECEDE model constructs Anxiety of nurses and PRECEDE model. J Health Syst Res. 2014;10(4):752-65.

35. Alavi Arjmand N, Kashaninia Z, Hosseini MA, Rezasoltani P. Effect of stress management on job stress and work-family conflict among nurses. Journal of hayat. 2013;18(4):81-91.

36. Vaghee S, Meshkin Yazd A, Asgharipour N, Ebrahimzadeh S. The effect of critical thinking training on nursesâ job stress in psychiatric ward. Journal of fundamentals of mental health. 2014;16(61):12-21.

37. Moayed MS, Mahmoudi H, Ebadi A, Salary MM, Danial Z. Effect of education on stress of exposure to sharps among nurses in emergency and trauma care wards. Trauma monthly. 2015 May;20(2):34-39

38. Gelsema TI, Van Der Doef M, Maes S, Akerboom S, Verhoeven C. Job Stress in the Nursing Profession: The Influence of Organizational and Environmental Conditions and Job Characteristics. International Journal of Stress Management. 2005;12(3):222.

39. Delvaux N, Razavi D, Marchal S, Brédart A, Farvacques C, Slachmuylder J-L. Effects of a 105 hours psychological training program on attitudes, communication skills and occupational stress in oncology: a randomised study. British journal of cancer. 2004;90(1):106-14.

4o. Phillips MR, Zhang J, Shi Q, Song Z, Ding Z, Pang $S$, et al. Prevalence, treatment, and associated disability of mental disorders in four provinces in China during 2001-05: an epidemiological survey. The Lancet. 2009;373(9680):2041-53.

41. Kawano Y. Association of job-related stress factors with psychological and somatic symptoms among Japanese hospital nurses: effect of departmental environment in acute care hospitals. Journal of occupational health. 2008;50(1):79-85.

42. Jones SC, Donovan RJ. Does theory inform practice in health promotion in Australia? Health Education Research. 2004;19(1):1-14.

43. Feqhi Farahmand N. Stress management for managers and employees. Journal of Management Perfection 2009(1);10:165-88

44. Hussein G. The Attitudes of Undergraduate Students Towards Motivation and Technology in a Foreign Language Classroom. International Journal of Learning and Teaching. 2010;2(2):14-24.

45. 14- Zhang G. Survey of occupational stress of secondary and elementary school teachers and the lessons learned. Chinese Educ Soc 2007;40(5):32-9.

46. Feqhi Farahmand N. Stress management for managers and employees. Journal of Management Perfection2009(1);10:165-88. 
Cite this article as:

Madmoli M, Madmoli Y, Madmoli M. The Most Important Educational Interventions for Reducing Occupational Stress Among Nurses Working in Health Centres: A Systematic

Source of support: Nil, Conflict of interest: None declared

Review Study. Int Healthc Res J. 2019;3(2):50-55. doi: 10.26440/IHRJ/0302.05.521076

\section{AUTHOR AFFILIATIONS:}

1. Emergency Medical Technician, Dezful University of Medical Sciences, Dezful, Iran

2. M.Sc. Student of Nursing, Student Research Committee, Ahvaz Jundishapur University of Medical Sciences, Ahvaz, Iran

3. Student Research Committee, Shoushtar faculty of Medical Sciences, Shoushtar, Iran

\section{*Corresponding Author:}

Mostafa Madmoli

Emergency Medical Technician

Dezful University of Medical Sciences

\section{Dezful, Iran}

